\title{
Integrated Physiological and Transcriptomic Analyses Reveal a Regulatory Network of Anthocyanin Metabolism Contributing to the Ornamental Value in a Novel Hybrid Cultivar of Camellia japonica
}

\author{
Liqin Pan ${ }^{1,2}$, Jiyuan Li ${ }^{1, *}$, Hengfu Yin ${ }^{1}$, Zhengqi Fan ${ }^{1, *}$ and Xinlei Li ${ }^{1}$ \\ 1 Research Institute of Subtropical Forestry, Chinese Academy of Forestry, Hangzhou 311400, China; \\ LiqinPAN@tzvcst.edu.cn (L.P.); hfyin@caf.ac.cn (H.Y.); lixinlei2020@caf.ac.cn (X.L.) \\ 2 Research Institute of Horticultural Botany, Taizhou Vocational College of Science \& Technology, \\ Taizhou 318020, China \\ * Correspondence: jiyuan_li@caf.ac.cn (J.L.); fzq_76@caf.ac.cn (Z.F.)
}

Received: 5 November 2020; Accepted: 2 December 2020; Published: 7 December 2020

check for updates

\begin{abstract}
Camellia japonica is a plant species with great ornamental and gardening values. A novel hybrid cultivar Chunjiang Hongxia (Camellia japonica cv. Chunjiang Hongxia, $\mathrm{CH}$ ) possesses vivid red leaves from an early growth stage to a prolonged period and is, therefore, commercially valuable. The molecular mechanism underlying this red-leaf phenotype in $\mathrm{C}$. japonica $\mathrm{cv}$. $\mathrm{CH}$ is largely unknown. Here, we investigated the leaf coloration process, photosynthetic pigments contents, and different types of anthocyanin compounds in three growth stages of the hybrid cultivar $\mathrm{CH}$ and its parental cultivars. The gene co-expression network and differential expression analysis from the transcriptome data indicated that the changes of leaf color were strongly correlated to the anthocyanin metabolic processes in different leaf growth stages. Genes with expression patterns associated with leaf color changes were also discussed. Together, physiological and transcriptomic analyses uncovered the regulatory network of metabolism processes involved in the modulation of the ornamentally valuable red-leaf phenotype and provided the potential candidate genes for future molecular breeding of ornamental plants such as Camellia japonica.
\end{abstract}

Keywords: Camellia japonica; leaf color; anthocyanin; metabolism; transcriptome

\section{Introduction}

Belonging to the Theaceae family, Camellia japonica is one of the ornamentally and economically most valuable flowering plants all over the world. Different from some Camellia species used as tea plants, such as Camellia sinensis, C. japonica is grown as a type of ornamental shrub mainly for its leaves and flowers [1]. The extensive cultivation of $C$. japonica as a houseplant for landscaping and gardening has kept increasing its economic value in China, Japan, Australia, the USA, and a number of European countries [2]. More than 23,000 C. japonica cultivars have been recorded [3], among which most cultivars appear with a red or dark red leaf color only for a short period from the sprouting to the emergence of leaves, and then quickly change to green [1,4]. The duration length of the red leaves in Camellia is a major factor for determining its ornamental value. Thereby, cultivation of red-leaf C. japonica has broad garden application prospects.

Leaf color changes in plants are associated with the relative content alteration of pigments, including chlorophyll, carotenoids, anthocyanins, and their glycosides [5-7]. It has been reported that the abundant anthocyanin biosynthesis in the leaves and the excessive accumulation of anthocyanin-associated metabolites in the vacuole led to a red-leaf phenotype in ornamental plants, 
including Quercus coccifera and Malus crabapples [8,9]. To date, the mechanism involved in the determination of leaf color in Camellia plants, especially in C. japonica, remains poorly understood. Ying et al. showed that in a Camellia cultivar Jinhua Beauty (C. japonica cv. Jinhua Beauty), total anthocyanins were accumulated, while the biosynthesis of colorless polyphenol was blocked during the red-leaf period [4]. In a purple-leaf Camellia sinensis cultivar Sunrouge (C. sinensis cv. Sunrouge), total anthocyanins were abundant in the buds and 30-day-old leaves. The metabolic intermediates of flavonoid biosynthesis in the purple-leaf cultivar were significantly higher than that of green-leaf cultivars of $C$. sinensis [10]. Flavonoids in the leaves of camellias plants, such as catechins and table catechins, exhibited dynamic amounts during different developmental and growth stages, which were correlated to the leaf color changes [11]. Recently, epicatechin glucose was proposed as an important substrate of procyanidins biosynthesis in five Camellia species. However, the biosynthesis processes of flavonoids including procyanidins in Camellia genus still lack sufficient studies [12].

In addition to analyses in physiological and metabolic levels, molecular bases of ornamentally valuable phenotypes of Camellia plants have attracted more attention. Nevertheless, most of the related studies were focused on morphological regulation of Camellia flowers, such as the shape of petals [13]. Recently, several groups investigated the transcriptional control of genes involved in metabolic regulation of leaves and buds of $C$. sinensis plants $[14,15]$. Genes involved in the flavonoid biosynthetic pathway were diversely influenced by shading, which caused modified natural products accumulation, and therefore, potentially affected the quality of the tea products [14]. However, no evidence regarding the gene regulatory network of leaf color change in C. japonica has been shown, and the molecular bases of the red-leaf phenotype of $C$. japonica plants need to be clarified.

In the present work, we report a novel hybrid Camellia cultivar Chunjiang Hongxia (Camellia japonica cv. Chunjiang Hongxia, $\mathrm{CH}$ ), which was generated using cultivars Black Magic (Camellia japonica cv. Black Magic, BM) and Black Opal (Camellia japonica cv. Black Opal, BO) as female and male parent, respectively. $\mathrm{CH}$ cultivar showed a longer red-leaf duration compared with $\mathrm{BM}$ and $\mathrm{BO}$. The dynamic changes of metabolites at different leaf growth stages in $\mathrm{CH}, \mathrm{BM}$, and $\mathrm{BO}$ were investigated. Comparative transcriptome analysis further uncovered the network of regulatory signaling pathways and key genes involved in the red-leaf phenotype of Camellia japonica plants.

\section{Materials and Methods}

\subsection{Plant Materials and Growth Condition}

Three Camellia japonica cultivars used in this study are Chunjiang Hongxia (Camellia japonica cv. Chunjiang Hongxia, CH), Black Magic (Camellia japonica cv. Black Magic, BM), and Black Opal (Camellia japonica cv. Black Opal, BO). BM is a cultivated variety of Camellia japonica $[1,16,17]$ and $\mathrm{BO}$ is a hybrid cultivar of Camellia japonica cv. Gem Bell and Camellia japonica cv. Black Tsubaki $[1,16,17]$.

On December 2016, five cutting seedlings with a consistent growth stage of each cultivar were grown in the greenhouse of the Research Institute of Subtropical Forestry of the Chinese Academy of Forestry ( $30^{\circ} 06^{\prime}$ North, $119^{\circ} 96^{\prime}$ East; Fuyang, Zhejiang, China). Investigation was started from March 2018. Each bud was labeled by germination, and the time of budding and the number of days of growth from budding were recorded. For measurements of pigments contents, ultra-performance liquid chromatography-tandem mass spectrometer (UPLC-MS/MS) experiments, as well as RNA-seq, sampling was repeated in randomly selected three plants for each cultivar, as a total of three biological replicates. For determination of pigments contents, the first, second, and third leaf on the top were harvested when 10, 30, and 50 days after leaf-expansion, respectively. The leaves were immediately placed in an ice box when harvested for the subsequent treatments. For UPLC-MS/MS tests and RNA-seq, the fourth, fifth, and sixth leaf on the top were harvested 10, 30, and 50 days, respectively. The leaves were immediately frozen in liquid nitrogen and ground to powder, then split to two aliquots. In the remaining two plants of each cultivar, three buds in total were randomly selected for leaf color 
measurements at each growth stage. Similar to the former tests, leaves when 10, 30, and 50 days after leaf-expansion were collected and used.

\subsection{Measurement of Leaf Color Indices and Contents of Pigments}

Leaf color was determined by handy NF555 spectrophotometers (Nippon Denshoku, Japan) using the method as previously described [18]. Three points at the leaf tip, leaf center, and petiole base of the leaf abaxial and adaxial surfaces were measured. The indices including $L^{*}, a^{*}$, and $b^{*}$ values were recorded, in which $L^{*}$ is the brightness of the color, ranging from 0 to 100 and the represented color range is from black (0) to white (100); $\mathrm{a}^{*}$ indicates the green-red axis, ranging from -120 to 120 , and the represented color range is from green (negative) to red (positive); $b^{*}$ indicates blue-yellow axis, ranging from -120 to 120 and the represented color range is from blue (negative) to yellow (positive).

Chlorophyll and carotenoids contents were measured using the method reported by Wellburn et al. [19]. In brief, absorbances of chlorophyll $\mathrm{a}$ and $\mathrm{b}$ and carotenoids were determined at $470 \mathrm{~nm}, 646 \mathrm{~nm}$, and $663 \mathrm{~nm}$, respectively, using 755 PC spectrophotometer (Shanghai Spectral Instrument, China). The contents were then calculated with the formulas:

$$
\begin{gathered}
\text { chlorophyll a content }=12.21 \mathrm{~A} 663-2.81 \mathrm{~A} 646 ; \\
\text { chlorophyll b content }=20.13 \mathrm{~A} 646-5.03 \mathrm{~A} 663 ; \\
\text { carotenoids content }=(1000 \mathrm{~A} 470-3.27 \mathrm{Ca}-104 \mathrm{Cb}) / 98 .
\end{gathered}
$$

The extraction of anthocyanins employed the method described by Kerio et al. [20]. The total anthocyanins contents were calculated using absorbances at $520 \mathrm{~nm}$ and $700 \mathrm{~nm}$ with a series of formulas:

$$
\begin{gathered}
\mathrm{A}=\mathrm{pH} 1.0(\mathrm{~A} 520-\mathrm{A} 700)-\mathrm{pH} 4.5(\mathrm{~A} 520-\mathrm{A} 700) ; \\
\mathrm{C}\left(\mathrm{mg} \cdot \mathrm{L}^{-1}\right)=(\mathrm{A} \times \mathrm{MW} \times \mathrm{DF} \times 1000) /(\varepsilon \times \mathrm{CW}) ; \\
\mathrm{MW}=449.2 \mathrm{~g} \cdot \mathrm{mol}^{-1} \\
\mathrm{DF}(\text { dilution factior })=10 \\
\varepsilon(\text { Molar Extinction Coefficient })=26900 \mathrm{~L} /(\mathrm{mol} \cdot \mathrm{cm}) \\
\mathrm{CW}(\text { cuvette width })=1 \mathrm{~cm} \\
\text { anthocyanins content }\left(\mathrm{mg} \cdot \mathrm{g}^{-1}\right)=(\mathrm{C} \times 0.01) / 0.1 .
\end{gathered}
$$

Each measurement was technically repeated three times, and the mean value was used as a biological replicate.

\subsection{Ultra-Performance Liquid Chromatography-Tandem Mass Spectrometer (UPLC-MS/MS)}

All samples were assayed by ACQUITY UPLC I-Class System equipped with an BEH $\mathrm{C}_{18}$ column $(1.7 \mu \mathrm{m} 100 \times 2.1 \mathrm{~mm}$ i.d.) coupled to a Xevo G2-XS QTOF mass spectrometer (Waters Corporation, Milford, MA, USA) as previously described [21]. In brief, around $0.5 \mathrm{~g}$ of leaf tissue powder was resuspended in $7.5 \mathrm{~mL}$ extraction buffer (15\% methanol, $0.5 \%$ formic acid) and sonicated for $15 \mathrm{~min}$. The slurry was centrifuged at $5000 \times g$ for $15 \mathrm{~min}$, and $6 \mathrm{~mL}$ of supernatant was saved as a test solution. The test solution was then diluted five times with methanol, and $90 \mathrm{uL}$ of the resulted solution was mixed with $10 \mu \mathrm{L}$ of internal standard. After centrifugation at $12,000 \times g$ for $10 \mathrm{~min}, 2 \mu \mathrm{L}$ of the supernatant was loaded onto UPLC-QTOF-MS. The quantitative analysis was performed based on the ratio of the peak area of the sample to the internal standard.

For chromatographic separation, the column was kept at $35^{\circ} \mathrm{C}$. The mobile phase A was $0.2 \%$ formic acid in Milli-Q water and phase B was acetonitrile. The linear gradient elution procedure was started at 10\% B for $1 \mathrm{~min}$, followed by $20 \%$ B for $3.5 \mathrm{~min}, 26 \%$ B for $5.5 \mathrm{~min}, 95 \%$ B for $21 \mathrm{~min}, 95 \%$ B 
for $25 \mathrm{~min}, 10 \% \mathrm{~B}$ for $25.1 \mathrm{~min}$, and maintenance for $30 \mathrm{~min}$. The flow rate was $0.4 \mathrm{~mL} \cdot \mathrm{min}^{-1}$ and the injected volume was $2 \mu \mathrm{L}$.

For MS analysis, the mass spectrometer equipped with an ESI source was controlled by MassLynx v 4.1 software (Waters Corporation, Milford, MA, USA). A full MS scan was operated in the range $m / z$ 50-1200 Da. The capillary voltage was $1 \mathrm{kV}$. The source temperature was $120^{\circ} \mathrm{C}$ and the desolvation temperature was $450{ }^{\circ} \mathrm{C}$. The cone gas flow was $50 \mathrm{~L} \cdot \mathrm{h}^{-1}$ and the desolvation gas flow was $800 \mathrm{~L} \cdot \mathrm{h}^{-1}$. Data-dependent acquisition (DDA) was applied for the secondary mass analysis and multiple reaction monitoring (MRM) was used for quantification.

\subsection{Statistics for Physiological Indices}

Statistical analyses for physiological indices were done using SPSS19.0 software (IBM, New York, NY, USA). A one-way ANOVA test was done for a comparison of the compound contents and a Pearson two-tailed test was used for the measurement of the correlation between pigment contents and leaf color parameters of three Camellia japonica cultivars.

\subsection{RNA-Seq and Data Processing}

The total RNA was extracted using RNAprep Pure Plant Kit (TIANGEN, Beijing, China). The amount and quality of RNA samples were measured by Nanodrop 2000 (Thermo Scientific, Waltham, MA, USA) and RNA Nano 6000 kit (Agilent Technologies, Santa Clara, CA, USA). Approximately $1 \mu \mathrm{g}$ of RNA was used for sequencing library construction using NEBNext Ultra II Directional RNA Library Prep Kit (New England Biolabs, Hitchin, UK) and barcoded libraries were loaded on Illumina HiSeq 2000 at Biomarker Technologies, Inc., Beijing, China. The RNA-seq data has been submitted to BioProject database of National Center for Biotechnology Information (NCBI) with the accession number PRJNA682688.

The raw reads were cleaned by FastQC [22] and trimmed by SolexaQA_v.2.5 [23]. Reads with more than $10 \% \mathrm{~N}$ or more than $50 \%$ low-quality bases $(\mathrm{Q}<5)$ were filtered. Trinity [24] was used to assemble contigs to unigenes. The resulted unigenes were aligned and annotated in five databases using BLASTX [25]: Swiss-Prot [26], COG [27], GO [28], NR [29], KEGG [30] (with E-value $<1.0 \times 10^{-5}$ ). Gene ontology (GO) annotation was obtained by BLAST2GO [31] and functional classification was performed by WEGO software [32]. KEGG pathway annotation was carried out using Blastall [33,34]. The expression levels of unigenes were calculated using RSEM [35] by fragments per kilobase per million (FPKM). The differential expression analysis was performed using DESeq2 [36]. Differentially expressed unigenes (DEUs) were defined with the criteria of false discovery rate (FDR) $<0.01$ and $\log _{2}$ (Fold Change) $\geq 1$.

\subsection{Quantitative Real-Time PCR ( $q P C R)$}

The reversed transcription was done using PrimeScript ${ }^{\mathrm{TM}} \mathrm{RT}$ reagent Kit with gDNA Eraser (Takara, Dalian, China) and qPCR was performed using TB Green Advantage qPCR Premix (Takara, Dalian, China) with incubated in $95^{\circ} \mathrm{C}$ for $30 \mathrm{~s}$, followed by 40 cycles of $95^{\circ} \mathrm{C}$ for $5 \mathrm{~s}$ and $60^{\circ} \mathrm{C}$ for $31 \mathrm{~s}$. The expression levels were calculated by $2^{-\Delta \Delta C T}$ method and NADPH was used as an internal control. The primers include: ANS-F, GCCAAAAGAAGAGCTGACGG; ANS-R, CCTTCTTCAACGCCTTCCGA; UFGT-F, TAACCCTTGGGCTAATCCGC; UFGT-R, TGTTGTCCGGGATTGGTGAG; GAPDH-F, GGGAATCCTT GGTTACACTGAG; GAPDH-R, ACCCCATTCGTTGTCATACC. Three biological replicates were applied for each gene.

\section{Results and Discussion}

\subsection{Characterization of the Red-Leaf Phenotype in Camellia japonica Cv. Chunjiang Hongxia}

Camellia japonica cv. Chunjiang Hongxia $(\mathrm{CH})$ was generated by a cross between $\mathrm{C}$. japonica cv. Black Magic (BM) as the female parent and C. japonica cv. Black Opal (BO) as the male parent (Figure 1A). 
Compared with $\mathrm{BM}$ and $\mathrm{BO}, \mathrm{CH}$ showed a bright red leaf color at 10 days after leaf expansion and sustained the dark red color for 30 days (Figure $1 \mathrm{~B}$ and Figure S1). While BM and BO only exhibited a weak red color in 10-day leaves, which had mostly turned to green at 30 days and 50 days (Figure 1B). Consistently, the quantification of leaf color indices demonstrated that the $L^{*}$ and $b^{*}$ values, representing the color range of black/white and blue/yellow, respectively, were comparatively stable across three leaf growth stages in all three cultivars, while the $\mathrm{a}^{*}$ value representing the color range of green/red was dramatically changed during the growth of the leaves (Figure 1C-E), which confirmed that the leaf color change during expansion was mainly in the color range of green/red. Moreover, the $\mathrm{a}^{*}$ values of $\mathrm{CH}$ in 10-day and 30-day leaves were positive, and significantly higher than BM and BO (Figure 1D). The 30-day and 50-day leaves of BM both had negative $\mathrm{a}^{*}$ values, while in $\mathrm{BO}$, the 30-day leaves were almost at the turning point of red to green and the 50-day leaves also had a negative $\mathrm{a}^{*}$ value. These results demonstrated that the red-leaf period of $\mathrm{CH}$ was more than one month, much longer than its parent cultivars. Interestingly, both $\mathrm{L}^{*}$ and $\mathrm{b}^{*}$ values in $\mathrm{CH}$ were lower than $\mathrm{BM}$ and $\mathrm{BO}$ in most of stages (Figure $1 \mathrm{C}, \mathrm{E}$ ), indicating that in addition to red, $\mathrm{CH}$ leaves contained more blue and dark materials than $\mathrm{BM}$ and $\mathrm{BO}$.

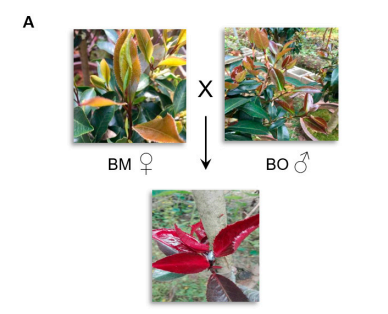

$\mathrm{CH}$
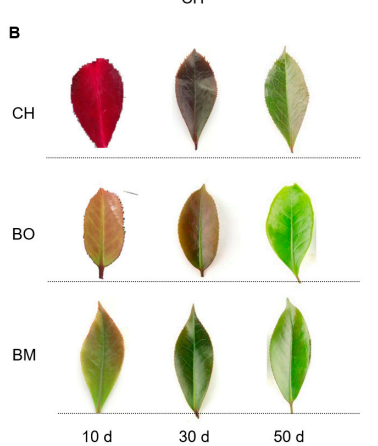

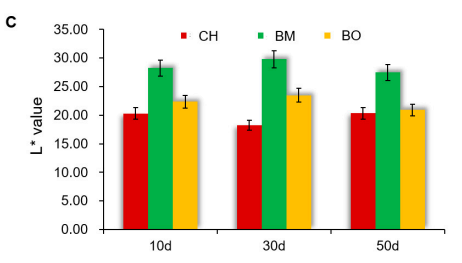

D
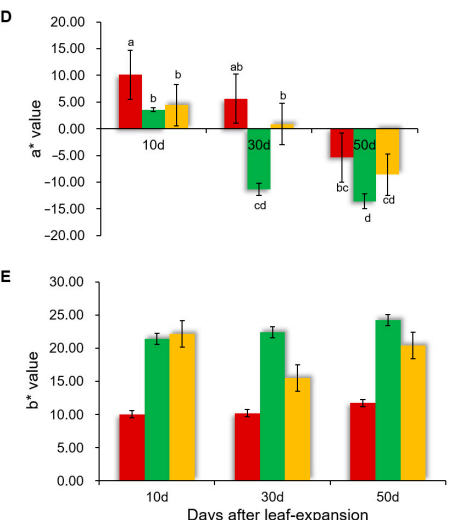

Figure 1. Characterization of leaf color in Camellia japonica cultivars. (A) Camellia japonica cv. Chunjiang Hongxia $(\mathrm{CH})$ is the offspring of $C$. japonica cv. Black Magic (BM) as the female parent and $C$. japonica cv. Black Opal (BO) as the male parent. Representatives of 10-day-old (10 d) leaves of each cultivar are shown. (B) Representatives of leaves of $\mathrm{CH}, \mathrm{BM}$, and $\mathrm{BO}$ cultivars 10 days, 30 days, and 50 days after leaf-expansion. (C-E) Leaf color indices of $\mathrm{CH}, \mathrm{BM}$, and $\mathrm{BO}$ cultivars at different leaf growth stages. $L^{*}(\mathbf{C}), a^{*}(\mathbf{D})$, and $b^{*}(\mathbf{E})$ values were used. $L^{*}$ represents a color range from 0 (black) to 100 (white); $\mathrm{a}^{*}$ represents a color range from -120 (green) to 120 (red); $\mathrm{b}^{*}$ represents a color range from -120 (blue) to 120 (yellow). Data are means \pm standard deviation (SD), $n=3$. Different letters above the bars indicate significant differences ( $p<0.05$, one-way ANOVA).

\subsection{Contents of Pigments in $\mathrm{CH}$ Leaves}

Pigments are key molecules in formation of the leaf colors [8,9]. Given that the color changes happened after 10 days, 30 days, and 50 days in leaves of $\mathrm{CH}, \mathrm{BM}$, and $\mathrm{BO}$, we measured the contents of anthocyanin, chlorophyll a, chlorophyll $\mathrm{b}$, and carotenoids in the three growth stages accordingly. The anthocyanin content of $\mathrm{CH}$ was significantly higher than that of $\mathrm{BM}$ and $\mathrm{BO}$ in 10-day and 30-day leaves, and it was decreased with leaf growth in all three cultivars (Figure 2A). In comparison, chlorophyll a, chlorophyll b, and carotenoids in the three cultivars showed similar levels at each leaf growth stage and exhibited a trend of increase when leaves were getting older 
(Figure 2B), indicating that these three pigments in C. japonica plants were accumulated with age in a cultivar-independent manner. Moreover, the proportion of each pigment was consistent with the leaf color alteration of $\mathrm{CH}, \mathrm{BM}$, and $\mathrm{BO}$ during different growth stages (Figure 2C). In 10-day leaves showing red color, anthocyanin had the highest level among the pigments, and its proportion reduced with age, while proportions of the other three pigments kept increasing. In line with the green-turning process of the leaf color in three cultivars, when the proportion of anthocyanin dropped below $40 \%$ in the 50-day leaves of $\mathrm{CH}$ and $\mathrm{BO}$ and in the 30-day and 50-day leaves of $\mathrm{BM}$, leaf color was turned from red to green. This association was further supported by a statistical correlation between color parameters and pigment contents (Table 1). The $a^{*}$ value, representing the color range of green/red, was positively correlated to anthocyanin contents in all three cultivars. In $\mathrm{CH}$, the $\mathrm{L}^{*}$ value representing the color range of black/white was negatively correlated to the contents of chlorophyll $\mathrm{a}$ and carotenoids, while chlorophyll $\mathrm{b}$ was negatively correlated to the $\mathrm{a}^{*}$ value. It has been reported that the abundancy of chlorophyll a, chlorophyll $b$, and carotenoids was associated with leaf darkening and green-turning in flowering plants [37]. On the other hand, the anthocyanin has been considered as a main component contributing to red color in leaves of higher plants [38]. In summary, our results demonstrated that the red-leaf phenotype was highly related to the proportion of anthocyanin in the entire pigment contents.
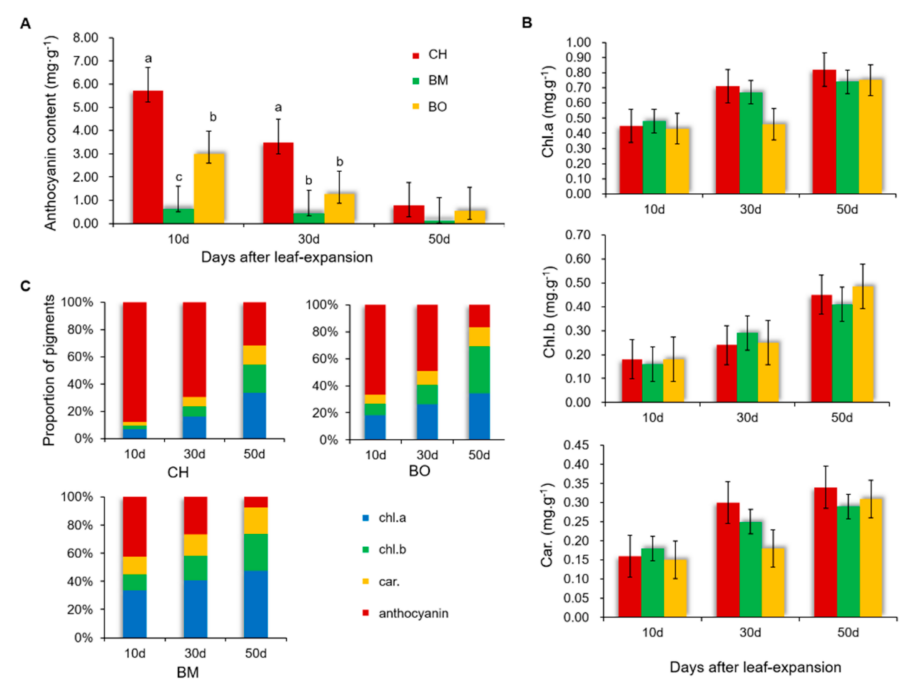

Figure 2. Contents of anthocyanins (A), chlorophyll a, chlorophyll b, and carotenoids (B) in Camellia japonica cultivars $\mathrm{CH}, \mathrm{BM}$, and $\mathrm{BO}$ leaves at different growth stages. (C) Proportion of chlorophyll a, chlorophyll b, carotenoids, and anthocyanins in $\mathrm{CH}, \mathrm{BM}$, and $\mathrm{BO}$ leaves at different growth stages. Data are means \pm standard deviation $(\mathrm{SD}), \mathrm{n}=3$. Different letters above the bars indicate significant differences ( $p<0.05$, one-way ANOVA).

Table 1. The correlation between pigment contents and leaf color parameters of three Camellia japonica cultivars. Pearson two-tailed test was used $\left({ }^{*} p<0.05,{ }^{* *} p<0.01\right)$.

\begin{tabular}{cccccc}
\hline Cultivars & $\begin{array}{c}\text { Leaf Color } \\
\text { Parameter }\end{array}$ & Chlorophyll a & Chlorophyll b & Anthocyanin & Carotenoid \\
\hline \multirow{3}{*}{$\mathrm{CH}$} & $L^{*}$ & $-0.999^{*}$ & -0.962 & 0.945 & $-0.999^{*}$ \\
& $a^{*}$ & -0.971 & $-1.000^{* *}$ & $0.999^{*}$ & -0.948 \\
& $b^{*}$ & 0.886 & 0.757 & -0.719 & 0.920 \\
$\mathrm{BM}$ & $L^{*}$ & -0.972 & -0.889 & 0.782 & -0.942 \\
& $a^{*}$ & -0.914 & -0.984 & $1.000^{*}$ & -0.951 \\
& $b^{*}$ & 0.899 & 0.772 & -0.631 & 0.849 \\
$\mathrm{BO}$ & $L^{*}$ & -0.735 & -0.722 & 0.971 & -0.794 \\
& $a^{*}$ & -0.906 & -0.898 & $0.998^{*}$ & -0.941 \\
& $b^{*}$ & 0.190 & 0.208 & 0.469 & 0.098 \\
\hline
\end{tabular}




\subsection{Dynamic Changes of Polyphenols and Anthocyanins in CH Leaves}

There are six common types of anthocyanins in plant tissues, including delphinidin, peonidin, petunidin, pelargonidin, cyanidin, and malvidin [39]. At present, 25 kinds of anthocyanins have been detected in the Camellia petals, among which the cyanidin group had a biggest proportion and delphinidin was also detected [40,41]. Only a few studies have been done on anthocyanin metabolism in C. japonica leaves, especially for specific quantification of each type of anthocyanins as well as the biosynthesis precursors. According to the key substances produced in the anthocyanin biosynthesis pathway [42,43], two kinds of polyphenols and six kinds of anthocyanins were selected for ultra-performance liquid chromatography-tandem mass spectrometer (UPLC-MS/MS) measurement in 10-day, 30-day and 50-day leaves of $\mathrm{CH}$ cultivar, and BM cultivar was used as a control. For two types of polyphenols, catechin and epicatechin contents in $\mathrm{CH}$ were close to or higher than that in $\mathrm{BM}$ at each growth stage. The amount of catechin was reduced with age in both $\mathrm{CH}$ and $\mathrm{BM}$, while epicatechin content was stable in $\mathrm{CH}$ but reduced in BM with age (Figure 3A). In 10-day leaves, these two contents did not show obvious differences. All six anthocyanins showed significantly higher amounts in $\mathrm{CH}$ than $\mathrm{BM}$ at all three stages (Figure 3B). Among them, malvidin, delphinidin, petunidin-3-o- $\beta$-glucoside, and cyanidin exhibited comparatively stable contents across growth stages in both cultivars. Additionally, the amount of these four types of anthocyanins sustained 50 days after leaf-expansion. Cyanidin-3-glucoside and pelargonidin-3-glucoside were significantly lower in older leaves compared with 10-day leaves. In $\mathrm{CH}$, the content of cyanidin-3-glucoside was $6.05 \mathrm{mg} \cdot \mathrm{g}^{-1}$ in 10-day leaves, which was the highest amount in all detected anthocyanins, and was in line with the total anthocyanin content detected by spectrophotometer (Figure 2A). It decreased by $87 \%$ in 50-day leaves compared with 10-day samples. The content of pelargonidin-3-glucoside was less than $0.07 \mathrm{mg} \cdot \mathrm{g}^{-1}$ in all leaves of $\mathrm{CH}$ and undetectable in BM. Hence, these two types of anthocyanins played a main role in the red-leaf phenotype of 10-day leaves in $\mathrm{CH}$.
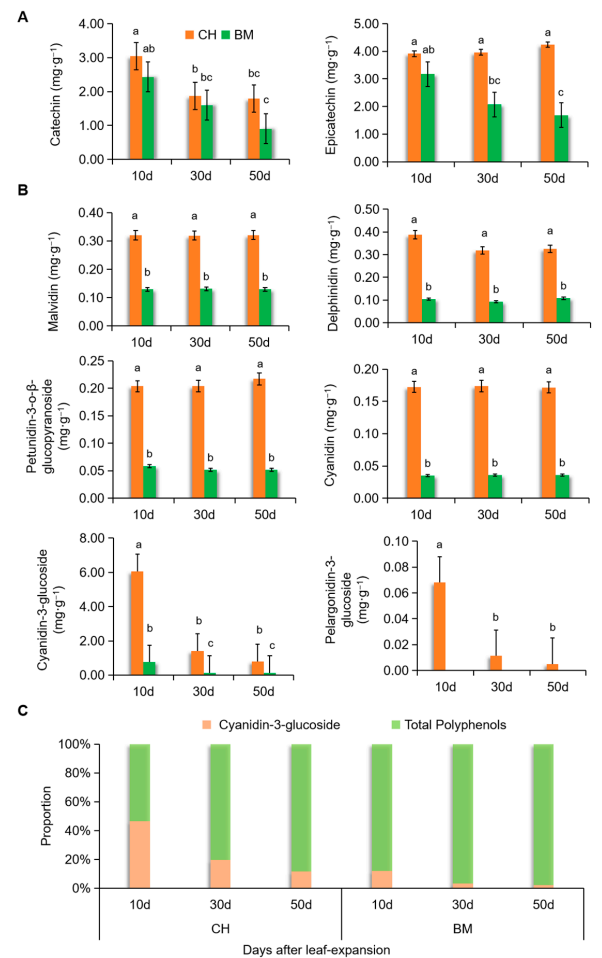

Figure 3. Contents of polyphenols including catechin and epicatechin (A) and anthocyanins including malvidin, delphinidin, petunidin-3-o- $\beta$-glucoside, cyanidin, cyanidin-3-glucoside, and pelargonidin-3glucoside (B) measured by UPLC-MS/MS. (C) Proportion of total polyphenols and cyanidin-3-glucoside in $\mathrm{CH}$ and $\mathrm{BM}$ leaves at different growth stages. Data are means \pm standard deviation (SD), $\mathrm{n}=3$. Different letters above the bars indicate significant differences ( $p<0.05$, one-way ANOVA). 
The anthocyanins affecting yellow and blue color in leaves, such as malvidin, delphinidin and petunidin-3-o- $\beta$-glucoside, showed stable contents across growth stages, which was consistent with the leaf color phenotype and the $\mathrm{a}^{*}$ value (Figure 1 ). The age-dependent reduction of cyanidin-3-glucoside, the main elicitor of red color in $\mathrm{CH}$ young leaves, was also the main reason of decrease of anthocyanin proportion in pigments due to its large amount. Furthermore, the ratio between the contents of cyanidin-3-glucoside and the sum of catechin and epicatechin was obviously higher in $\mathrm{CH}$ than $\mathrm{BM}$ at each stage, and dropped quickly with age in $\mathrm{CH}$ (Figure 3C), which was in line with the leaf color changes from red to green and turning to dark.

\subsection{Transcriptome Assembling and Annotation}

Transcriptome analysis was widely used for the investigation on the molecular basis of metabolic dynamics in plants [44]. We conducted RNA-seq with triplicates on 10-day, 30-day and 50-day leaf tissues of $\mathrm{C}$. japonica cultivars $\mathrm{CH}, \mathrm{BM}$, and $\mathrm{BO}$, respectively. After raw data filtering, more than 20 million reads were obtained per sample on average (Table S1). Replicates from one condition showed a high correlation to each other, indicating the reproductivity of this assay (Figure S2). A total of 54,189 unigenes were assembled from all clean reads, with a total length of 95,948,909 bp and an average length of $1770 \mathrm{bp}$ for each. Among these, 33,929 unigenes were longer than $1 \mathrm{~kb}$ and 19,251 were longer than $2 \mathrm{~kb}$. There were 42,931 unigenes annotated through BLASTX using the databases including Swiss-Prot, COG, GO, NR, and KEGG (Table S2). More than $76 \%$ of the annotated unigenes for each sample were longer than $1 \mathrm{~kb}$. The values of fragments per kilobase per million (FPKM) were employed to determine the transcription level of unigenes (Figure 4). Those with FPKM $\geq 0.5$ were considered as transcribed unigenes. Based on the FPKM of the transcribed unigenes from 27 libraries with unigenes of FPKM over 0.5, PCA analysis showed the similarity between $\mathrm{CH}$ and BM samples at the same growth stage. For instance, 10-day samples (T01, T02, and T03) of $\mathrm{CH}$ were close to 10-day samples (T10, T11, and T12) of BM, and 30-day (T04, T05, and T06-1) and 50-day (T07, T08, and T09) samples of $\mathrm{CH}$ were overlapped with 30-day (T13, T14, and T15) and 50-day (T16, T17, and T18) samples of BM, respectively. Intriguingly, BO samples were not overlapped with $\mathrm{CH}$ or BM samples at the same growth stage. Again, all biological replicates were in the same group, which confirmed the reproducible sampling (Figure $4 \mathrm{~A}$ ). The numbers of transcribed unigenes across all leaf samples were ranged from 26,518 to 33,754 (Figure $4 B$ ). In all three cultivars, there were more transcribed unigenes in 10-day leaves than that in older leaves. The unigenes with FPKM from the range of 0.5 to 5 and 5 to 100 had nearly the same proportion. Less than $5 \%$ of unigenes expressed a high level of FPKM, i.e., above 100. Most of the identified unigenes were transcribed across all three growth stages (Figure 4C). The 10-day-specific unigenes were five to nine times more than those at the other two stages in three cultivars, suggesting active transcription at early growth stage of leaves.

\subsection{Co-Expression Module Analysis of Transcribed Unigenes}

The weighted gene co-expression network analysis (WGCNA) can classify genes with similar expression patterns as modules and connect these modules of co-expressed genes to certain phenotypes [45]. We applied WGCNA to 27 RNA-seq samples including leaf tissues from three C. japonica cultivars of $\mathrm{CH}, \mathrm{BM}$, and $\mathrm{BO}$ at three growth stages and co-expression modules were constructed. Unigenes of FPKM $<0.5$ were removed and a total of 7268 transcribed unigenes were clustered into 23 co-expression modules (Figure S3A). Modules highly correlated to samples could reveal unigenes with expression patterns potentially involved in red-leaf phenotype (Figure S3B). The modules of MEcyan, MEdarkgreen, and MElavendarblush3 were significantly correlated to 10-day samples of $\mathrm{CH}$, 30-day samples of $\mathrm{CH}$ and 10-day samples of $\mathrm{BM}$ with a correlation coefficient of $0.77\left(p=2 \times 10^{-7}\right), 0.82\left(p=5 \times 10^{-9}\right)$, and $0.72\left(p=2 \times 10^{-5}\right)$. These three modules reflected differences of co-expressed unigenes between early and late stages in $\mathrm{CH}$ and between $\mathrm{CH}$ and BM cultivars, and therefore, were selected for further KEGG pathways enrichment analysis. In the modules connected to 10-day samples of both $\mathrm{CH}$ and $\mathrm{BM}$, phenylpropanoid biosynthesis pathway 
was enriched, while in 30-day samples, this pathway was not identified but glutathione metabolism and $A B C$ transporter pathways were enriched (Figure S4). The upstream substrates required for anthocyanin and flavonoid biosynthesis are derived from the phenylalanine metabolism pathway and the accumulation of flavonoids is directly related to the ABC transporter and glutathione metabolism process [14,42]. Thus, co-expression module analysis using transcribed unigenes implied that the metabolism pathways of phenylpropanoid biosynthesis were associated with red leaf color in the early growth stage of C. japonica plants.

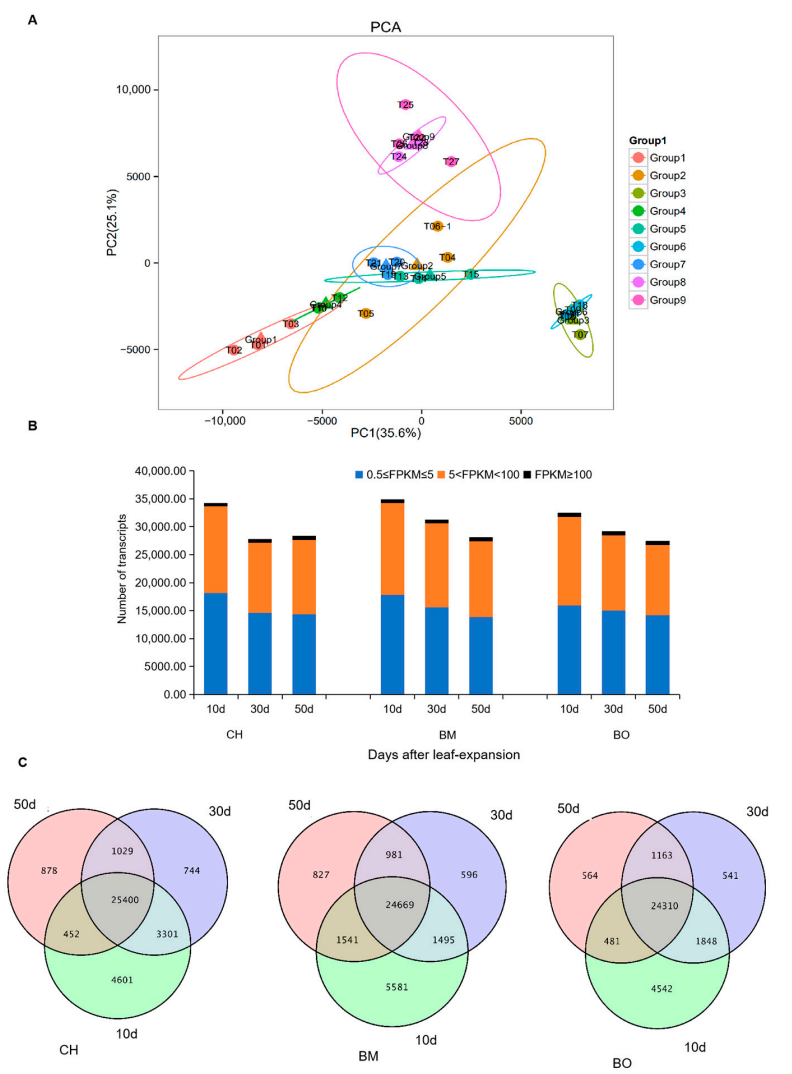

Figure 4. Characterization of unigene expression. (A) PCA analysis of 27 RNA-seq samples based on unigene transcription levels. The key of the sample code can be found in Table S1. (B) Distribution of unigene transcription levels in $\mathrm{CH}, \mathrm{BM}$, and $\mathrm{BO}$ leaves at different growth stages. (C) Venn diagram showing the overlap of the transcribed unigenes among different growth stages in $\mathrm{CH}, \mathrm{BM}$, and $\mathrm{BO}$ leaves.

3.6. Differential Expression Analysis Reveals a Regulatory Network of Anthocyanin Metabolism Related to Red-Leaf Phenotype

In addition to co-expression analysis, transcriptome data allowed direct comparisons between conditions related to phenotypes [46]. To further investigate key regulators involved in red-leaf phenotype of $\mathrm{CH}$, differential expression analysis was performed. Differentially expressed unigenes (DEUs) were identified using the threshold of false discovery rate $(F D R)<0.01$ and Log2(Fold Change) $\geq 1$. Among the leaf growth stages, in older leaf samples there were more unigenes up-regulated than down-regulated compared with 10-day leaf tissues in all three cultivars (Table S3). Consistently, more DEUs were detected in the comparison between 50-day and 10-day leaf samples than that between 30-day and 10-day samples. In CH, there were 11,130 DEUs between 50-day and 10-day samples and 8692 DEUs between 30-day and 10-day samples, respectively. Among cultivars, the DEU numbers between parent cultivars and $\mathrm{CH}$ were larger than that between $\mathrm{BM}$ and $\mathrm{BO}$, as expected (Table S4). Comparatively, the difference between $\mathrm{BO}$ and $\mathrm{CH}$ was bigger than that between $\mathrm{BM}$ and $\mathrm{CH}$. There were $8556 \mathrm{DEUs}$ between BM and $\mathrm{CH}$ and 12,401 DEUs between $\mathrm{BO}$ and $\mathrm{CH}$. 
Gene ontology (GO) analysis was carried out for DEUs from comparisons between 50-day or 30-day leaf tissues and 10-day samples of $\mathrm{CH}$, and comparisons between 10-day samples of $\mathrm{BM}$ or $\mathrm{BO}$ and $\mathrm{CH}$. GO terms identified in all these comparisons were similarly enriched in metabolic process for biological process and catalytic activity for molecular function, which was in line with the severely changing anthocyanin biosynthesis and metabolism (Figures S5 and S6). Moreover, KEGG pathway analysis on these DEUs showed a high enrichment on phenylpropanoid biosynthesis in the comparisons between the older leaf tissues and 10-day samples of $\mathrm{CH}$, which confirmed the co-expression module outputs (Figure 5A,B). Between the 10-day samples of $\mathrm{BM}$ and $\mathrm{CH}$, the pathways of phenylalanine, tyrosine, and tryptophan biosynthesis were also enriched, while $\mathrm{BO}$ showed changes of photosynthesis pathway compared with $\mathrm{CH}$ (Figure 5C,D). In summary, pathway enrichment identified DEUs mainly related to modulation of upstream metabolites of anthocyanin biosynthesis, such as phenylpropanoid or phenylalanine.
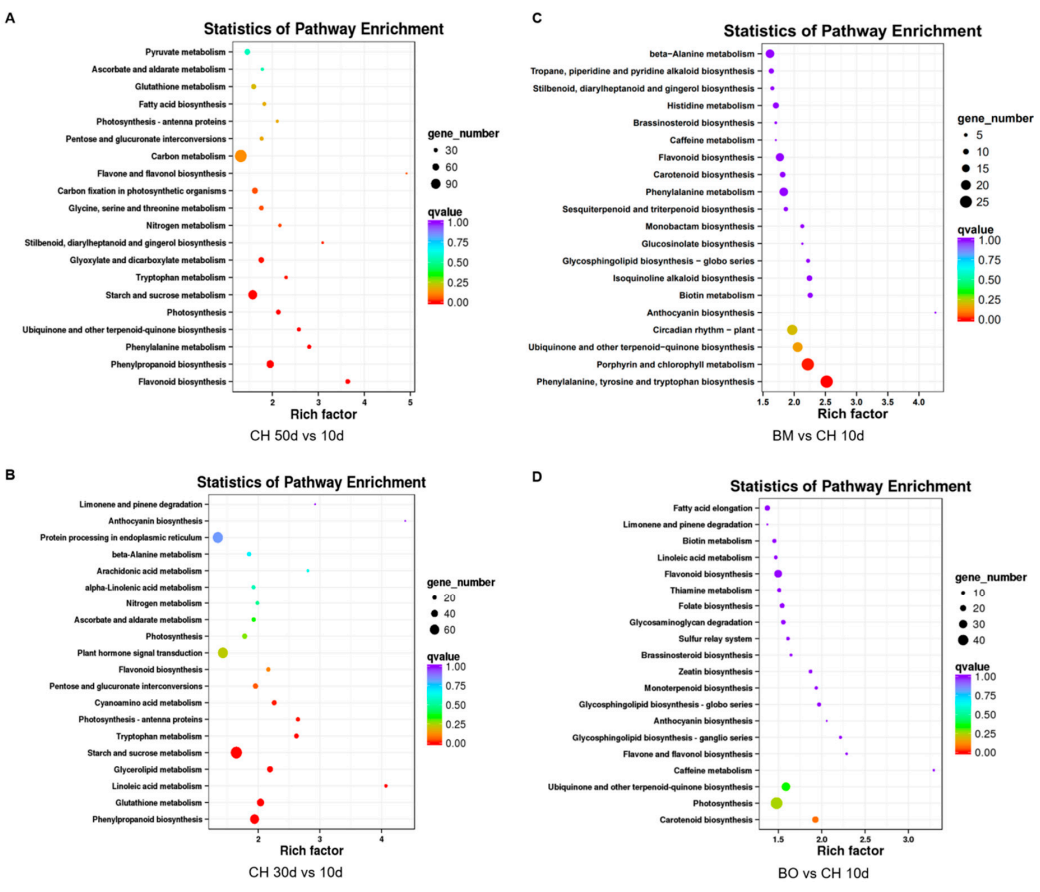

Figure 5. Enrichment of KEGG pathways of differentially expressed unigenes (DEUs) in transcriptome comparisons between 50-day and 10-day leaves of $\mathrm{CH}(\mathrm{A})$, between 30-day and 10-day leaves of $\mathrm{CH}$ (B), between 10-day leaves of BM and $\mathrm{CH}(\mathbf{C})$, and between 10-day leaves of BO and $\mathrm{CH}(\mathbf{D})$.

The DEUs associate with anthocyanin metabolism were further listed for different comparisons (Figure 6). Among unigenes with significantly higher expression levels in 10-day leaf tissues of $\mathrm{CH}$ compared with other conditions, BMK_Unigene_207848, BMK_Unigene_092871, and BMK_Unigene_032609 were detected in at least two comparisons. BMK_Unigene_207848 and BMK_Unigene_092871 were aligned and annotated as ANS, the anthocyanidin synthase gene, or the leucoanthocyanidin dioxygenase gene, in C. japonica plants. ANS plays a key role in anthocyanins biosynthesis and catalyzes the conversion of colorless leucoanthocyanidins to anthocyanidins, which elicits critical effects on plant coloration [47]. BMK_Unigene_032609 was annotated as UFGT, the UDP-glucose:anthocyanidin 3-O-glucosyltransferase gene in C. japonica plants. UFGT has been considered to be another critical enzyme in anthocyanin biosynthesis, which catalyzes the synthesis of cyanidin 3-glucoside in the presence of UDP-glucoses and cyanidins, producing the first stable anthocyanin metabolites [48]. UFGT directly affects the biosynthesis and accumulation of plant anthocyanins and can have a decisive influence on the color of plant tissues [49]. The expression levels of ANS and UFGT were much lower in 10-day samples of BM and $\mathrm{BO}$ compared with $\mathrm{CH}$, and both dramatically decreased after 10 days post leaf-expansion in $\mathrm{CH}$, demonstrating that these two genes were crucial positive regulators of 
anthocyanin abundance in leaves at early growth stage (Figure 7). The transcription levels of ANS and UFGT were verified by quantitative real-time PCR and their expression variations detected in RNA-seq were consistent with qPCR.
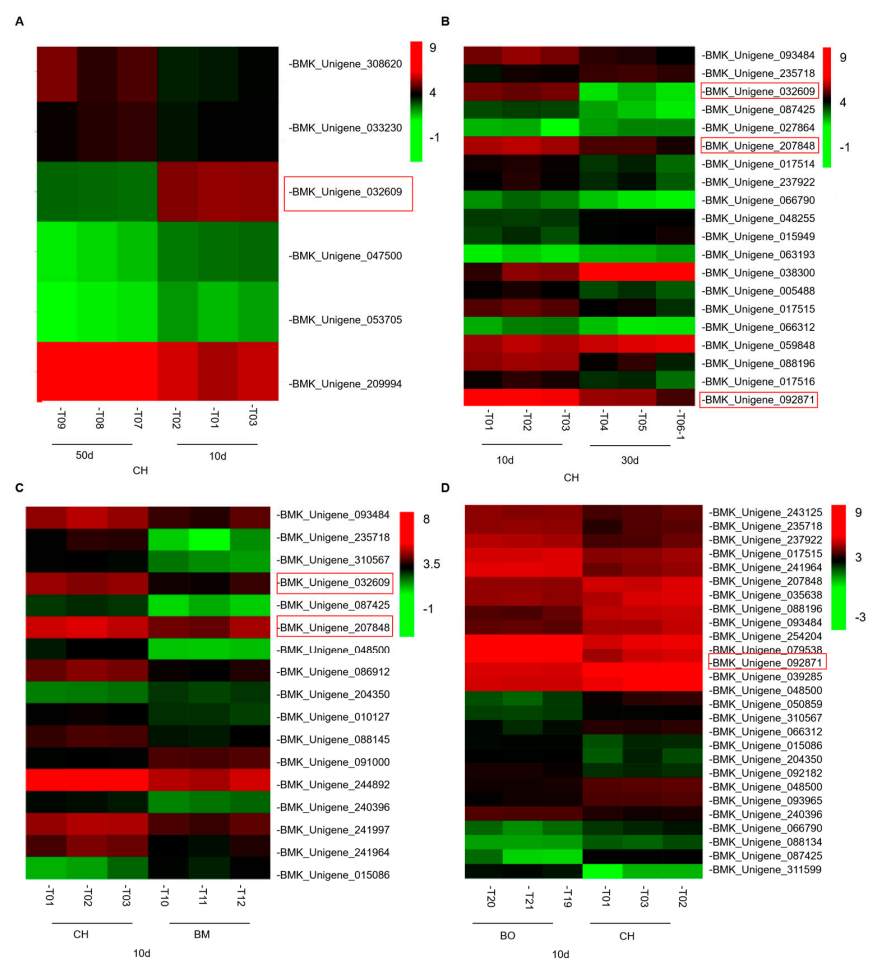

Figure 6. Heatmap showing anthocyanin metabolic signaling associated unigenes that were differentially expressed between 50-day and 10-day leaves of $\mathrm{CH}$ (A), between 30-day and 10-day leaves of $\mathrm{CH}$ (B), between 10-day leaves of $\mathrm{BM}$ and $\mathrm{CH}(\mathbf{C})$, and between 10-day leaves of $\mathrm{BO}$ and $\mathrm{CH}(\mathbf{D})$. Red frames show the unigenes of UFGT and ANS.
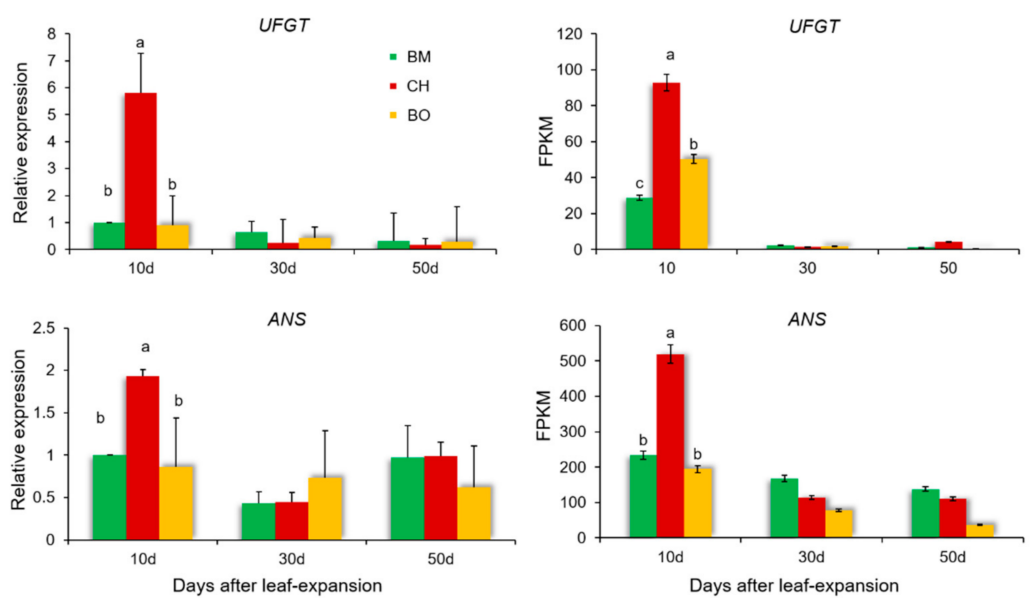

Figure 7. Verification of RNA-seq data using qPCR. The transcription levels of Camellia japonica genes UFGT and ANS in RNA-seq are indicated by fragments per kilobase per million (FPKM). The qPCR data are means \pm standard deviation (SD), $\mathrm{n}=3$. Different letters above the bars indicate significant differences (for qPCR, $p<0.05$, one-way ANOVA; for RNA-seq, FDR $<0.05$ ).

Together, the molecular basis of leaf color alteration from red to green and the anthocyanin content reduction in $\mathrm{CH}$ involved genes modulating phenylpropanoid biosynthesis pathway and anthocyanins accumulation. ANS and UFGT were two of the core functional regulators. 


\section{Conclusions}

Camellia japonica cv. Chunjiang Hongxia $(\mathrm{CH})$ is a novel $\mathrm{C}$. japonica cultivar with great ornamental values. $\mathrm{CH}$ shows a longer red-leaf period and a better quality of red leaf color compared with its parental cultivars. The red phenotype of $C$. japonica plants is mainly based on the ratio of contents of total anthocyanins to polyphenols. Cyanidin-3-glucoside is the primary contributor to the red leaf color in $\mathrm{CH}$. Moreover, transcriptome analysis indicates a high correlation between the phenylpropanoid biosynthesis pathway and anthocyanins accumulation as well as red-leaf phenotype. ANS and UFGT are key genes in the formation of the red-leaf phenotype in $\mathrm{CH}$. Together, this study reveals the physiological and molecular basis of the red-leaf phenotype of Camellia japonica cv. Chunjiang Hongxia, thereby providing a perspective in the improvement of the cultivation of novel ornamental Camellia japonica cultivars.

Supplementary Materials: The following are available online at http://www.mdpi.com/2223-7747/9/12/1724/s1, Figure S1: Representatives of leaves 10 days, 30 days, and 50 days after expansion in $\mathrm{CH}$ cultivar plants; Figure S2: Heat map showing Pearson's correlation between any two of 27 RNA-seq samples; Figure S3: Co-expression modules constructed by WGCNA for 27 RNA-seq samples. (A) Clustering dendrograms of unigenes with dissimilarity based on topological overlap. Modules are assigned different colors. (B) Heat map showing module-trait correlation. Each row corresponds to one module and each column to one sample as a trait. Each cell shows the correlation and p-value; Figure S4: Enrichment of KEGG pathways of unigenes in modules of MEcyan (A), MEdarkgreen (B) and MElavendarblush3 (C), respectively. Figure S5: The GO functional classification of unigenes differentially expressed between 50-day and 10-day leaves of $\mathrm{CH}(\mathrm{A})$, and between 30-day and 10-day leaves of $\mathrm{CH}$ (B). Figure S6: The GO functional classification of unigenes differentially expressed between 10-day leaves of BM and $\mathrm{CH}$ (A), and between 10-day leaves of BO and CH (B). Table S1: Reads numbers of RNA-seq samples; Table S2 Number of functionally annotated unigenes; Table S3: Numbers of differentially expressed unigenes in comparisons between growth stages; Table S4: Numbers of differentially expressed unigenes in comparisons between cultivars.

Author Contributions: L.P., J.L., and Z.F. designed the workflow; L.P. performed the experiments and data analysis; J.L., H.Y., Z.F., and X.L. interpreted the data and wrote the manuscript. All authors approved the submitted version of manuscript.

Funding: This research was funded by the national key R \& D program of China (2019YFD1001000).

Acknowledgments: The authors would like to thank people from the Li Lab and Fan Lab of the Research Institute of Subtropical Forestry for their kind help to our study.

Conflicts of Interest: The authors declare no conflict of interest.

\section{References}

1. Savige, T.J. The International Camellia Register; The International Camellia Society: Wirlinga, Australia, 1993.

2. Vela, P.; Salinero, C.; Sainz, M. Phenological growth stages of Camellia japonica. Ann. Appl. Biol. 2013, 162, 182-190. [CrossRef]

3. Wang, Z.; Shen, Y.; Short, P.; Yamaguchi, S.; Bergamini, D.; Salinero, C.; Beard, D.; Powell, J.; Corneo, A. The progress of international camellia register and development of camellia cultivar dictionary. In Proceedings of the International Camellia Society Congress, Nantes, France, 24-29 March 2018; pp. 24-33.

4. Ying, Z.; Zhang, J.; Ying, H.; Fan, Z.; Ni, S.; Wu, B.; Li, J. Content change of main chemical component relating to the red leaf of bud mutation Camellia variety 'Jinhua Meinü'. Acta Hortic. Sin. 2017, 44, 723-732.

5. Li, Y.; Zhang, Z.; Wang, P.; Wang, S.; Ma, L.; Li, L.; Yang, R.; Ma, Y.; Wang, Q. Comprehensive transcriptome analysis discovers novel candidate genes related to leaf color in a Lagerstroemia indica yellow leaf mutant. Genes Genomics 2015, 37, 851-863. [CrossRef]

6. Li, C.-F.; Xu, Y.-X.; Ma, J.-Q.; Jin, J.-Q.; Huang, D.-J.; Yao, M.-Z.; Ma, C.-L.; Chen, L. Biochemical and transcriptomic analyses reveal different metabolite biosynthesis profiles among three color and developmental stages in 'Anji Baicha' (Camellia sinensis). BMC Plant Biol. 2016, 16, 195. [CrossRef]

7. Yang, Y.; Chen, X.; Xu, B.; Li, Y.; Ma, Y.; Wang, G. Phenotype and transcriptome analysis reveals chloroplast development and pigment biosynthesis together influenced the leaf color formation in mutants of Anthurium andraeanum 'Sonate'. Front. Plant Sci. 2015, 6, 139. [CrossRef]

8. Karageorgou, P.; Manetas, Y. The importance of being red when young: Anthocyanins and the protection of young leaves of Quercus coccifera from insect herbivory and excess light. Tree Physiol. 2006, 26, 613-621. [CrossRef] 
9. Lu, Y.; Zhang, M.; Meng, X.; Wan, H.; Zhang, J.; Tian, J.; Hao, S.; Jin, K.; Yao, Y. Photoperiod and shading regulate coloration and anthocyanin accumulation in the leaves of malus crabapples. Plant Cell Tissue Organ Cult. 2015, 121, 619-632. [CrossRef]

10. Nesumi, A.; Ogino, A.; Yoshida, K.; Taniguchi, F.; Yamamoto, M.; Tanaka, J.; Murakami, A. 'Sunrouge', a New Tea Cultivar with High Anthocyanin. Jpn. Agric. Res. Q. 2012, 46, 321-328. [CrossRef]

11. Zhang, L.-Q.; Wei, K.; Cheng, H.; Wang, L.-Y.; Zhang, C.-C. Accumulation of catechins and expression of catechin synthetic genes in Camellia sinensis at different developmental stages. Bot. Stud. 2016, 57. [CrossRef]

12. Zhang, L.; Tai, Y.; Wang, Y.; Meng, Q.; Yang, Y.; Zhang, S.; Yang, H.; Zhang, Z.; Li, D.; Wan, X. The proposed biosynthesis of procyanidins by the comparative chemical analysis of five Camellia species using LC-MS. Sci. Rep. 2017, 7, 46131. [CrossRef]

13. Li, X.; Li, J.; Fan, Z.; Liu, Z.; Tanaka, T.; Yin, H. Global gene expression defines faded whorl specification of double flower domestication in Camellia. Sci. Rep. 2017, 7, 1-12. [CrossRef] [PubMed]

14. Liu, L.; Li, Y.; She, G.; Zhang, X.; Jordan, B.; Chen, Q.; Zhao, J.; Wan, X. Metabolite profiling and transcriptomic analyses reveal an essential role of UVR8-mediated signal transduction pathway in regulating flavonoid biosynthesis in tea plants (Camellia sinensis) in response to shading. BMC Plant Biol. 2018, 18, 233. [CrossRef] [PubMed]

15. Zhou, Q.; Chen, Z.; Lee, J.; Li, X.; Sun, W. Proteomic analysis of tea plants (Camellia sinensis) with purple young shoots during leaf development. PLoS ONE 2017, 12. [CrossRef] [PubMed]

16. Gao, J.; Chen, S.; Xu, B. The World's Best Camellia Cultivars; Zhejiang Science and Technology Publishing House: Hangzhou, China, 1998.

17. Gao, J.; Su, Y.; Hu, X. The Identification and Appreciation of the World's Outstanding Camellias; Zhejiang Science and Technology Publishing House: Hangzhou, China, 2007.

18. Wang, L.-S.; Shiraishi, A.; Hashimoto, F.; Aoki, N.; Shimizu, K.; Sakata, Y. Analysis of petal anthocyanins to investigate flower coloration of Zhongyuan (Chinese) and Daikon Island (Japanese) tree peony cultivars. J. Plant Res. 2001, 114, 33-43. [CrossRef]

19. Wellburn, A.R. The spectral determination of chlorophyll-a and chlorophhyll-b, as well as total carotenoids, using various solvents with spectrophotometers of different resolution. J. Plant Physiol. 1994, 144, 307-313. [CrossRef]

20. Kerio, L.; Wachira, F.; Wanyoko, J.; Rotich, M. Characterization of anthocyanins in Kenyan teas: Extraction and identification. Food Chem. 2012, 131, 31-38. [CrossRef]

21. Wang, X.; Zeng, L.; Liao, Y.; Zhou, Y.; Xu, X.; Dong, F.; Yang, Z. An alternative pathway for the formation of aromatic aroma compounds derived from L-phenylalanine via phenylpyruvic acid in tea (Camellia sinensis (L.) O. Kuntze) leaves. Food Chem. 2019, 270, 17-24. [CrossRef]

22. Cock, P.J.A.; Fields, C.J.; Goto, N.; Heuer, M.L.; Rice, P.M. The Sanger FASTQ file format for sequences with quality scores, and the Solexa/Illumina FASTQ variants. Nucleic Acids Res. 2010, 38, 1767-1771. [CrossRef]

23. Cox, M.P.; Peterson, D.A.; Biggs, P.J. SolexaQA: At-a-glance quality assessment of Illumina second-generation sequencing data. BMC Bioinform. 2010, 11. [CrossRef]

24. Grabherr, M.G.; Haas, B.J.; Yassour, M.; Levin, J.Z.; Thompson, D.A.; Amit, I.; Adiconis, X.; Fan, L.; Raychowdhury, R.; Zeng, Q.; et al. Full-length transcriptome assembly from RNA-Seq data without a reference genome. Nat. Biotechnol. 2011, 29, 644-652. [CrossRef]

25. Altschul, S.F.; Madden, T.L.; Schäffer, A.A.; Zhang, J.; Zhang, Z.; Miller, W.; Lipman, D.J. Gapped BLAST and PSI-BLAST: A new generation of protein database search programs. Nucleic Acids Res. 1997, 25, 3389-3402. [CrossRef] [PubMed]

26. Apweiler, R.; Bairoch, A.; Wu, C.; Barker, W.; Boeckmann, B.; Ferro, S.; Gasteiger, E.; Huang, H.; Lopez, R.; Magrane, M.; et al. UniProt: The Universal Protein knowledgebase. Nucleic Acids Res. 2004, 32, D115-D119. [CrossRef] [PubMed]

27. Tatusov, R.L.; Galperin, M.Y.; Natale, D.A.; Koonin, E.V. The COG database: A tool for genome-scale analysis of protein functions and evolution. Nucleic Acids Res. 2000, 28, 33-36. [CrossRef] [PubMed]

28. Ashburner, M.; Ball, C.A.; Blake, J.A.; Botstein, D.; Butler, H.; Cherry, J.M.; Davis, A.P.; Dolinski, K.; Dwight, S.S.; Eppig, J.T.; et al. Gene Ontology: Tool for the unification of biology. Nat. Genet. 2000, 25, $25-29$. [CrossRef] [PubMed]

29. Deng, Y.; Li, J.; Wu, S.; Zhu, Y.; Chen, Y.; He, F. Integrated NR database in protein annotation system and its localization. Comput. Eng. 2006, 32, 71-72. 
30. Kanehisa, M.; Goto, S.; Kawashima, S.; Okuno, Y.; Hattori, M. The KEGG resource for deciphering the genome. Nucleic Acids Res. 2004, 32, D277-D280. [CrossRef]

31. Conesa, A.; Götz, S.; García-Gómez, J.M.; Terol, J.; Talón, M.; Robles, M. Blast2GO: A universal tool for annotation, visualization and analysis in functional genomics research. Bioinformatics 2005, 21, 3674-3676. [CrossRef]

32. Ye, J.; Fang, L.; Zheng, H.; Zhang, Y.; Chen, J.; Zhang, Z.; Wang, J.; Li, S.; Li, R.; Bolund, L. WEGO: A web tool for plotting GO annotations. Nucleic Acids Res. 2006, 34, W293-W297. [CrossRef]

33. Kanehisa, M.; Goto, S. KEGG: Kyoto Encyclopedia of Genes and Genomes. Nucleic Acids Res. 2000, 28, 27-30. [CrossRef]

34. Rismani-Yazdi, H.; Haznedaroglu, B.Z.; Bibby, K.; Peccia, J. Transcriptome sequencing and annotation of the microalgae Dunaliella tertiolecta: Pathway description and gene discovery for production of next-generation biofuels. BMC Genomics 2011, 12, 148. [CrossRef]

35. Li, B.; Dewey, C.N. RSEM: Accurate transcript quantification from RNA-Seq data with or without a reference genome. BMC Bioinform. 2011, 12, 323. [CrossRef] [PubMed]

36. Anders, S.; Huber, W. Differential expression analysis for sequence count data. Genome Biol. 2010, 11, 1-12. [CrossRef] [PubMed]

37. Luo, J.; Shi, Q.; Niu, L.; Zhang, Y. Transcriptomic Analysis of Leaf in Tree Peony Reveals Differentially Expressed Pigments Genes. Molecules 2017, 22, 324. [CrossRef] [PubMed]

38. Gonzalez, A.; Zhao, M.; Leavitt, J.M.; Lloyd, A.M. Regulation of the anthocyanin biosynthetic pathway by the TTG1/bHLH/Myb transcriptional complex in Arabidopsis seedlings. Plant J. 2008, 53, 814-827. [CrossRef]

39. Kong, J.-M.; Chia, L.-S.; Goh, N.-K.; Chia, T.-F.; Brouillard, R. Analysis and biological activities of anthocyanins. Phytochemistry 2003, 64, 923-933. [CrossRef]

40. Li, J.-B.; Hashimoto, F.; Shimizu, K.; Sakata, Y. Anthocyanins from red flowers of Camellia cultivar 'Dalicha'. Phytochemistry 2008, 69, 3166-3171. [CrossRef]

41. Li, J.-B.; Hashimoto, F.; Shimizu, K.; Sakata, Y. Chemical taxonomy of red-flowered wild Camellia species based on floral anthocyanins. Phytochemistry 2013, 85, 99-106. [CrossRef]

42. Holton, T.A.; Cornish, E.C. Genetics and biochemistry of anthocyanin biosynthesis. Plant Cell 1995, 7, 1071-1083. [CrossRef]

43. Shimada, N.; Sasaki, R.; Sato, S.; Kaneko, T.; Tabata, S.; Aoki, T.; Ayabe, S.-I. A comprehensive analysis of six dihydroflavonol 4-reductases encoded by a gene cluster of the Lotus japonicus genome. J. Exp. Bot. 2005, 56, 2573-2585. [CrossRef]

44. Wang, F.; Dong, Y.X.; Tang, X.Z.; Tu, T.L.; Zhao, B.; Sui, N.; Fu, D.L.; Zhang, X.S. Comparative Transcriptome Analysis Revealing the Effect of Light on Anthocyanin Biosynthesis in Purple Grains of Wheat. J. Agric. Food Chem. 2018, 66, 3465-3476. [CrossRef]

45. Baker, R.L.; Leong, W.F.; Brock, M.T.; Rubin, M.J.; Markelz, R.J.C.; Welch, S.; Maloof, J.N.; Weinig, C. Integrating transcriptomic network reconstruction and eQTL analyses reveals mechanistic connections between genomic architecture and Brassica rapa development. PLoS Genet. 2019, 15. [CrossRef] [PubMed]

46. Wei, D.; Liu, M.; Chen, H.; Zheng, Y.; Liu, Y.; Wang, X.; Yang, S.; Zhou, M.; Lin, J. INDUCER OF CBF EXPRESSION 1 is a male fertility regulator impacting anther dehydration in Arabidopsis. PLoS Genet. 2018, 14. [CrossRef] [PubMed]

47. Jaakola, L. New insights into the regulation of anthocyanin biosynthesis in fruits. Trends Plant Sci. 2013, 18, 477-483. [CrossRef] [PubMed]

48. Hu, C.; Gong, Y.; Jin, S.; Zhu, Q. Molecular analysis of a UDP-glucose: Flavonoid 3-O-glucosyltransferase (UFGT) gene from purple potato (Solanum tuberosum). Mol. Biol. Rep. 2011, 38, 561-567. [CrossRef] [PubMed]

49. Wu, X.; Gong, Q.; Ni, X.; Zhou, Y.; Gao, Z. UFGT: The Key Enzyme Associated with the Petals Variegation in Japanese Apricot. Front. Plant Sci. 2017, 8. [CrossRef]

Publisher's Note: MDPI stays neutral with regard to jurisdictional claims in published maps and institutional affiliations. 